\title{
Athena: A Macintosh-Based Interactive Karyotyping System
}

\author{
Lucas J. van Vliet, Ian T. Young, Ton K. ten Kate, Brian H. Mayall, \\ Frans C.A. Groen, Roelof Roos
}

\begin{abstract}
In this article we describe a system we have constructed that provides for automated karyotyping of metaphase spreads. The software system Athena based upon the Macintosh II computer and a Data Translation's frame grabber - is written entirely in $\mathrm{C}$ and consists of approximately 200 Kbytes of executable code. While Athena does not provide (at this time) facilities for automated metaphase finding, it does provide facilities for automated image segmentation into individual chromosomes, automated measurements on each banded chromosome, and automated classification into the standard Paris-convention karyotype. Further, the system provides the ability to construct one or more chromosome data bases to represent the types of metaphase spreads and staining techniques that may be used in a given laboratory.

Because we believe that it is impossible to construct a system that can achieve perfect segmentation, perfect separation of touching and overlapping chromosomes, perfect localization of the centromeres, and perfect classification, the system offers the possibility for interaction at each of the above stages using the well-accepted Macintosh user interface.

The processing time for the complete analysis of a $512^{2}$ image that contains a metaphase spread, interphase nuclei, debris and so forth depends heavily on the 'quality' of the metaphase image. Depending upon the number of interactions required to complete the segmentation, the total processing time varies from less than 3 minutes for nice metaphase spreads to about 6 minutes when many chromosomes need to be separated manually using the mouse. Of that time 90 seconds represents the automated processing and the rest the time for human interaction. This is on a personal computer that uses a MC-68020 processor with a clock frequency of $16 \mathrm{MHz}$ and a MC-68881 floating point co-processor.
\end{abstract}

L.J. van Vliet, I.T. Young, T.K. ten Kate, B.H. Mayall, F.C.A. Groen, R. Roos, Athena: A MacintoshBased Interactive Karyotyping System, in: Automation of Cytogenetics, C. Lundsteen \& J. Piper (eds), Springer-Verlag, 1989, 47-66. 


\section{Introduction}

For more than 25 years medical scientists, physical scientists, mathematicians, engineers, and computer scientists have attempted to automate the analysis of metaphase chromosome spreads. In an important sense automated karyotyping was one of the first problems in digital image analysis and pictorial pattern recognition. That there are still a number of on-going and healthy research efforts in this field - approximately 25 years after the publication of Ledley [1964, 1965] and Neurath et al [1965]- shows that the problem is both exquisitely difficult and extremely important. It is important to remember, however, that it was less than 35 years ago that the correct number of chromosomes in the normal human chromosome complement, 46, was first enumerated [Tjio and Levan, 1956]. Further, the accurate and reproducible identification of each of the 24 possible chromosome classes by cytogenetic experts was only possible after the development of the banding stains by Caspersson and his co-workers [1970a, 1970b].

Thirty years ago digital image processing was in its infancy. Computers were located in inaccessible computation centers. Frame grabbers did not exist. Computer languages were limited to assembly language. A few simple measurements were being performed [Schreiber, 1956; Deriugen 1957]. The first laboratory-oriented computer was introduced in the early 1960's with the PDP-1 and the first collection of languages appeared at about the same time. As of this writing (1988) the computational power, memory, and storage capacity available 30 years ago in computation centers has been substantially surpassed by the power available in a desk-top personal computer that is priced at a level that makes it affordable to individual researchers and their families. The software sophistication available on all fronts - operating systems, user interfaces, languages, and image processing algorithms - has witnessed a comparable improvement [Duin, 1983; Krusemark and Haralick, 1983; Preston, 1983; Tamura et al, 1983].

With respect to the problem of automated karyotyping, the implications of these improvements have become evident. As of this writing (1988) more than 10 companies are producing systems for the computer processing of digitized chromosome images. These systems range from those that can only be described as "electronic scissors" to systems that combine metaphase finding as well as pattern classification of banded chromosomes to achieve a result that requires a bare minimum of human interaction.

In this same context, three of us (Young, Groen, Mayall) have been involved for more than 15 years in research into various aspects of the quantitative and automated analysis of digitized chromosome images. This research has covered virtually all aspects of the quantitative analysis of chromosome images:

- Metaphase finding [Bishop and Young, 1977]

- Automatic focussing [Mendelsohn and Mayall, 1972; Groen et al, 1985] 
- Analysis of banding patterns [Granlund et al, 1976; Visser, 1981; ten Kate et al, 1983]

- Centromere location [Visser, 1981; de Muinck Keizer, 1984]

- Chromosome aberrations [Mayall at al, 1974; Zack et al, 1976; Mayall at al, 1977a; Mayall at al, 1977b]

- Accurate DNA measurements from digital images [Mendelsohn et al, 1969; Mendelsohn et al, 1973; Mendelsohn et al, 1974; Groen and van der Ploeg, 1978; Mayall et al, 1984]

- DNA species within chromosomes [Young et al, 1983].

On the basis of these developments we decided in 1983 that we were in a position to construct a karyotyping workstation. With the exception of automated metaphase finding we had assembled the components required for automatically producing a karyogram given a metaphase cell. These components consisted of:

1) image segmentation to find the individual objects;

2) procedures for eliminating non-chromosome-like objects;

3) procedures to separate touching chromosomes;

4) an accurate method for chromosome rotation;

5) an accurate procedure for centromere determination;

6) an intuitive but quantitative way to describe the banding patterns, and;

7) a context-sensitive classification procedure based upon the length, centromeric index, and the aforementioned band description.

These components - together with a window/menu user interface - were implemented (in C) as a complete system on a Vicom image processing system and formed the Master's thesis research of ten Kate[1985]. As a system the Vicom was appropriate for this development effort but as a long-term solution it was inappropriate. The system was too expensive, the operating system clumsy, the $\mathrm{C}$ compilers marginal, and floating point calculations excruciatingly slow. To remedy this situation we decided in the Spring of 1987 to "port" the karyotyping package to an Apple Macintosh II personal computer. This system offered an inexpensive, powerful platform for the chromosome project. Aside from the $16 \mathrm{MHz}$ MC-68020 processor, high-speed hard disk, multi-megabyte RAM and MC-68881 floating point co-processor, the system also has an integrated 8-bit deep display and a professional software development environment, MPW [APDA, 1988] offering C, Pascal, object-oriented Pascal and assembly language.

The combination of our existing software, the Macintosh II platform and recently available frame grabbers has led to the development of Athena, a Macintosh-based interactive karyotyping system. The minimum Macintosh II configuration required for running the package consists of 4 Mbytes of RAM, a 40 Mbyte Hard Disk, a video expansion kit to provide 256 colors/grey levels on the screen, keyboard and mouse, and a color monitor. To provide hard copy, a Postscript-compatible laserprinter is necessary. In 
the remainder of this article we shall describe the software system as well as the preliminary results that have been achieved using the techniques contained in Athena.

\section{Using Athena}

Athena - much like a sister program Acuity developed for cell analysis on the Macintosh [Young and Roos, 1988] - is organized around the concept of an experiment. The user initiates a session with the program by "double-clicking" (or opening) the program icon shown in Figure 1. Thus the user initiates and interacts with the program along the lines defined in the Macintosh user protocol [Apple, 1985-1987].
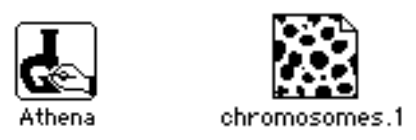

Figure 1: Left - The icon for the Athena program. Right - The icon for an image

After starting the program the user is offered a number of menu choices as shown in Figure 2.

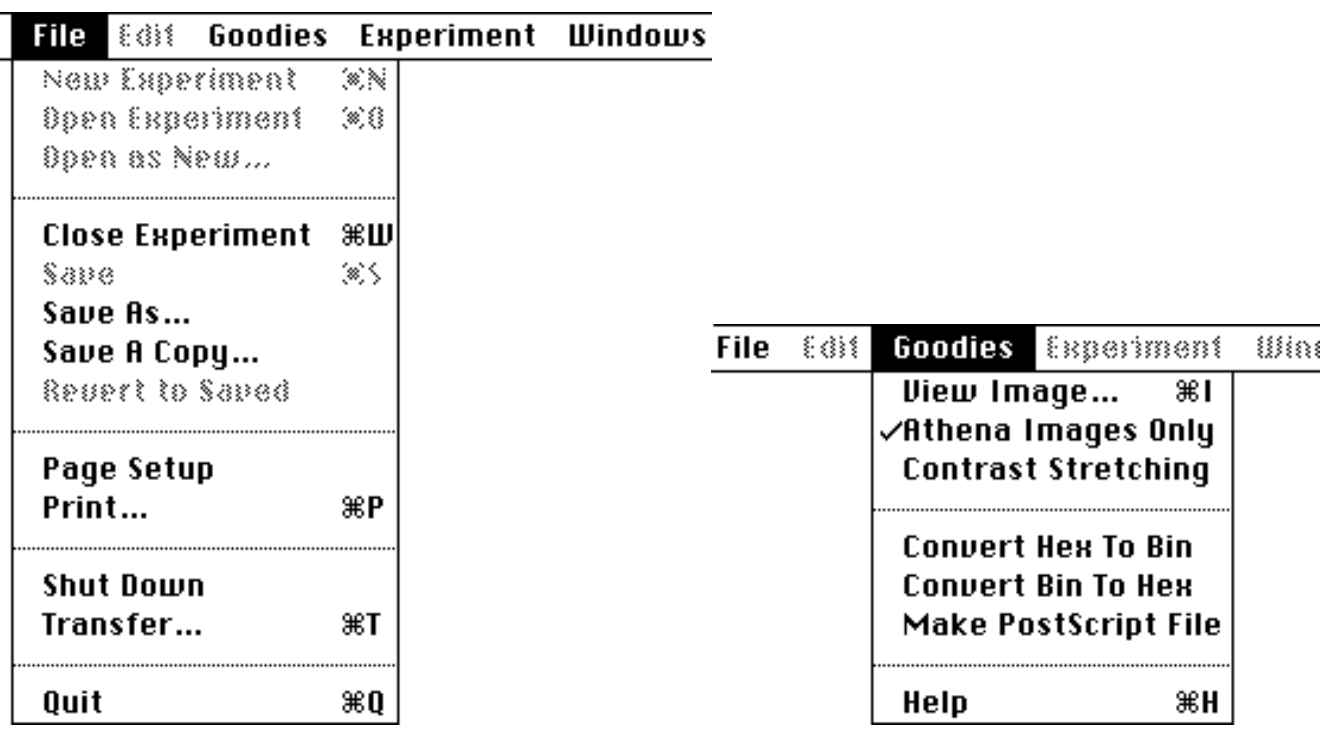

Figure 2: Left - The File menu that is used to define/open an experiment. Right - The Goodies menu that is used to preview an image, adjust the display contrast, or reformat image data.

The File menu - aside from several administrative functions - offers the user the ability to start a new experiment or to continue a previous one. The other possible choice after 
starting the program is the Goodies menu that allows the user to import, reformat, or display an image.

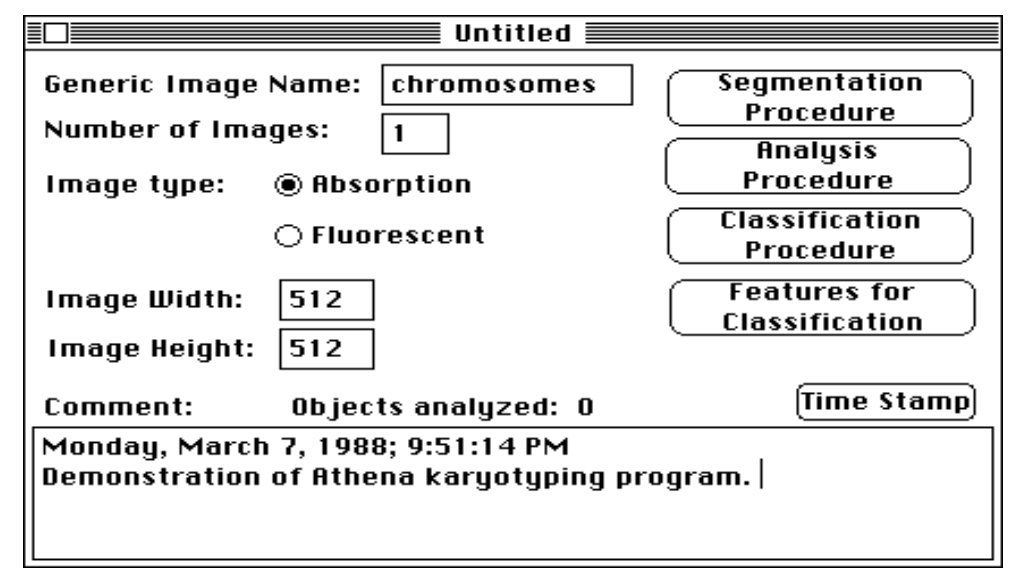

Figure 3: Experiment window associated with an - as yet - Untitled experiment. By clicking on the buttons Segmentation, Analysis, Classification and Features, the user can adjust the parameters associated with the various phases of the program.

After opening an old experiment (or starting a new one) the user is presented with a window as shown in Figure 3. By filling in the various entries in this window and the four sub-windows -Segmentation, Analysis, Classification, Features - the user defines the parameters, data sets, and so forth that are used when the program is executed. Arbitrary text as well as the current date and time can be entered in the comment block.

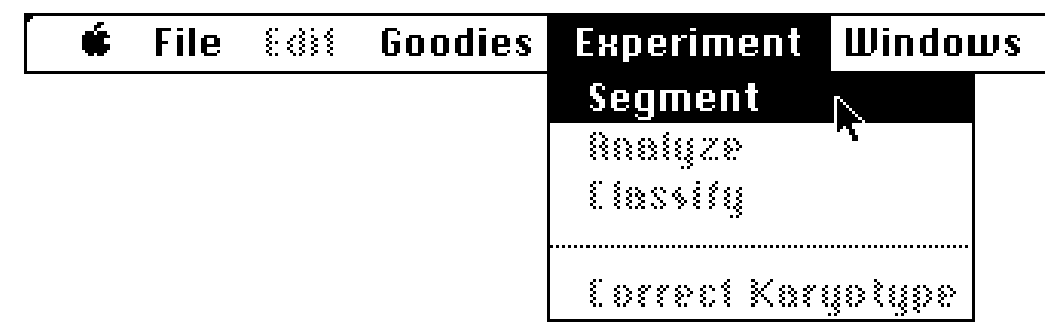

Figure 4: Menu choices associated with the various sequential steps in the karyotyping process.

At each of the three stages in the processing of the metaphase spread the user is presented with the opportunity to interact with the program. The three stages are intended to be used sequentially and are illustrated in Figure 4.

In the following sections we shall discuss each of these stages and the menu choices (as depicted in Figure 3) that are associated with them. 


\section{Segmentation}

The fundamental technique used for segmentation is a combination of shading correction, thresholding, and binary image operations. Shading correction is offered as an option to remove (possible) effects associated with camera sensitivity or the illumination conditions. Shading correction is accomplished through the use of the grey-level morphologic filtering operations dilation (maximum) and erosion (minimum). The estimate of the background shading $\mathbf{S}$ from an original image $\mathbf{I}$ is given by:

$$
\mathbf{S}=\operatorname{Min}_{\mathbf{n}}\left(\operatorname{Max}_{\mathbf{n}}(\mathbf{I})\right)
$$

where $\mathbf{n}$ is the neighborhood (support) of the filter. In this case the filter is always square of size $\mathbf{n}$ and the value of $\mathbf{n}$ should be chosen to be larger than the diameter of the largest object (usually an interphase nucleus) to be found in the image. The corrected image is then given by:

$$
\mathrm{C}=\mathrm{WHITE}+(\mathbf{I}-\mathrm{S})
$$

where WHITE is simply the maximum possible value (usually 255) in the absorption image.

Thresholding is used to convert the image into a binary representation where the chromosomes and other objects are black (1) and the background is white (0). The various algorithms used to choose the threshold are displayed in the Segmentation window (Figure 5). The threshold selection algorithms will not be described here; the user is instead referred to Young and Roos [1988], Ridler and Calvard [1978], and Vossepoel et al [1979]. 


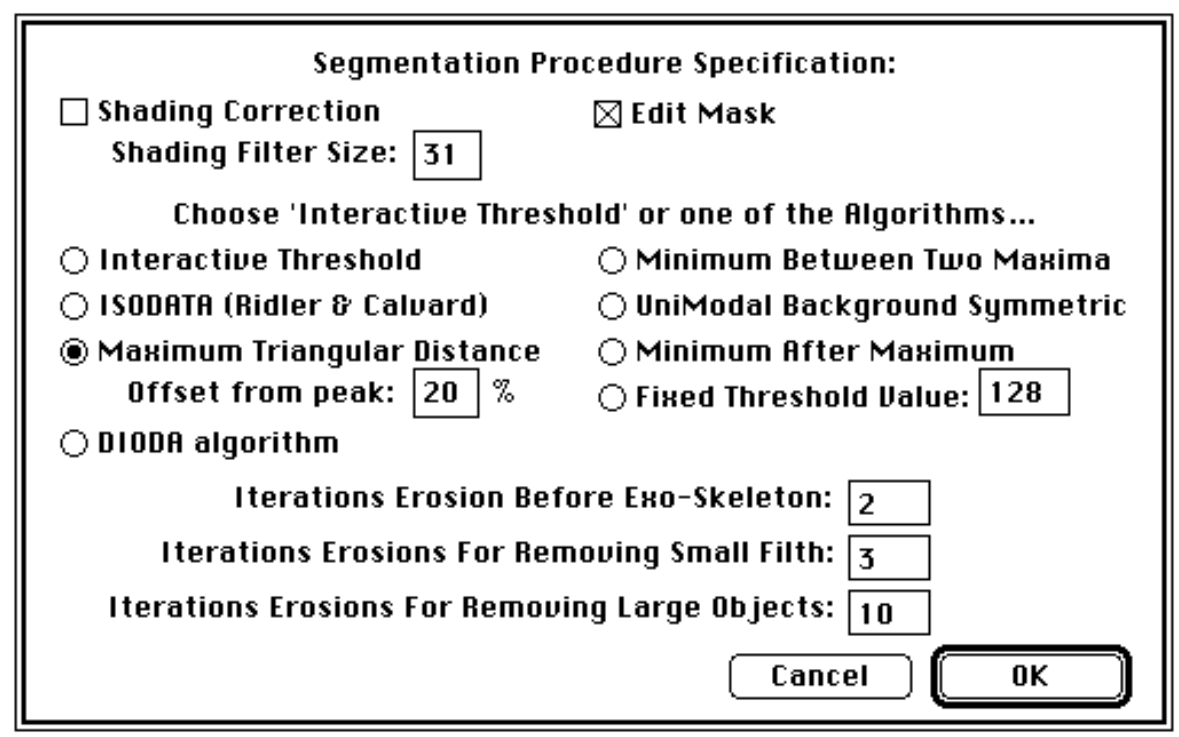

Figure 5: Choosing Segmentation Procedure offers the user the opportunity to specify the parameters associated with shading correction, thresholding, and binary image filtering. In addition, the user can indicate whether he/she wishes to correct interactively the segmentation result before the second stage - analysis - begins.

Binary image filtering is used to reduce the inevitable "false positives" and "false negatives" produced by thresholding. The operations erosion, dilation, propagation, exclusive-or, skeletonizing, and anding are used to eliminate small artefacts and large objects, to fill holes inside chromosomes, and to separate touching chromosomes. The actual algorithms used to implement these operations are based upon the recently developed techniques of Van Vliet and Verwer [1988].

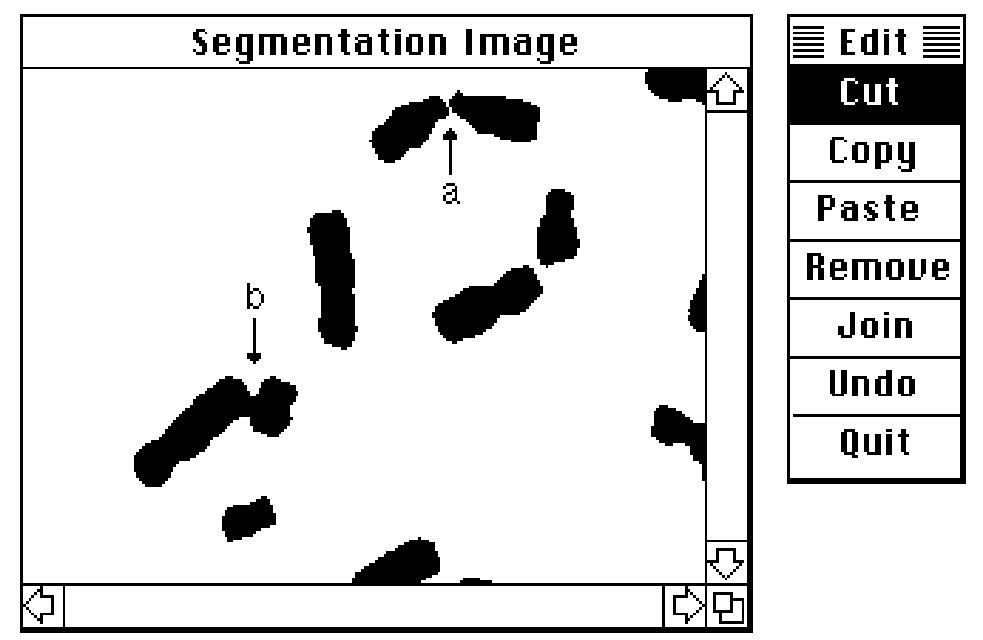

Figure 6: Near end of the segmentation phase, the user is offered the opportunity to correct errors if Edit Mask has been chosen (see Figure 5). 
At the completion of the segmentation phase the user can correct the remaining errors through the use of the mouse and a menu interface (Figure 6).

We try to separate slightly touching chromosomes by a few erosions after which the background skeleton (exo-skeleton) is computed. This exo-skeleton forms dividing lines to separate the touching chromosomes. At point $a$ in Figure 6, the exo-skeleton operation has successfully separated the two touching chromosomes. At point $b$, however, too few erosion passes (see Figure 5) were used to separate the chromosomes. By choosing Cut from the "pop-up" menu, the user can separate the two chromosomes. In the actual Macintosh display the user sees the grey-level image with contrast-stretching to maximize the visibility of details. The presumed chromosome borders are outlined in red. The mouse-based interactive procedure is straightforward. At the end of the segmentation phase each object - presumably a single chromosome - has been found, labeled and counted.

\section{Analysis}

The analysis phase consists of 1) rotating the individual chromosomes so that their long axis is parallel to the y-coordinate in the image [Groen et al, 1976], 2) determining the centromere position using the technique developed by Visser [1981] and 3) measuring the chromosome length, the centromeric index, and the band descriptors as described by Visser [1981]. The parameters associated with this phase are shown in Figure 7. This window is activated by selecting Analysis Procedure (see Figure 3).

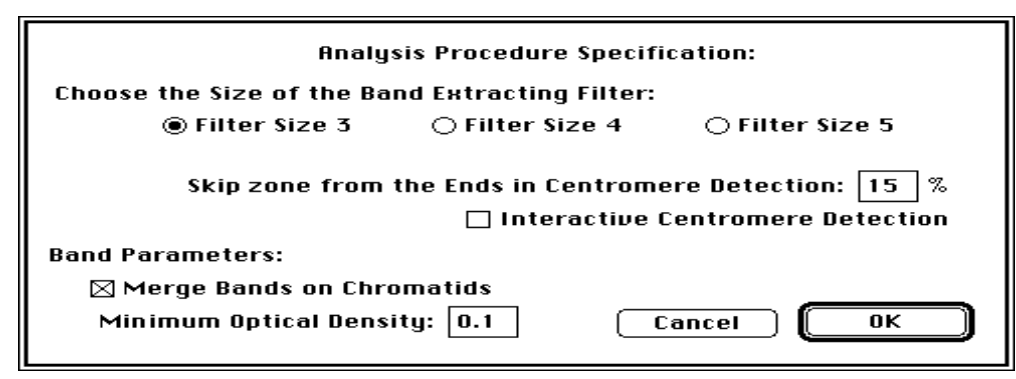

Figure 7: The parameter window associated with the analysis phase. 


\subsection{Measuring the centromeric index}

We define the centromeric index as:

$$
\mathbf{C I}=\frac{\text { Length of the short arm }(\mathrm{P} \text { terminal })}{\text { Total length of chromosome }} \times 1000
$$

This produces a number in the interval $0<\mathbf{C I}<500$. Automatic centromere detection has proven to be a difficult task and none of the techniques available achieves $100 \%$ success. Many techniques are based upon a search for a pair of concavities along the chromosome contour. Pairs of opposite concavities then form candidates for the centromere position. Athena's automatic centromere detector searches for two points having the shortest distance between the left and the right contour of a chromosome. In order to avoid detection at the ends of the chromosomes (telomeres), a certain distance from the top and bottom of the chromosomes is skipped. The user specifies this value as a percentage. The default value of $15 \%$ approaches the centromeric index of the acrocentric chromosomes of classes 13 through 15 .

As of this writing the interactive correction of centromeric position has not yet been implemented. The interface will resemble that of "Fat-Bits" except with grey levels. That is, the user will be presented with an enlarged, grey-level image of the chromosomes that he/she chooses for centromeric editing. A "pop-up" menu such as that shown in Figure 6 will then guide the user.

\subsection{Measuring the band parameters}

Assuming absorption imagery, bands are considered as dark regions of the chromosome. An optical density-based threshold selects the dark parts as regions potentially bearing a band. In this way the detection of vague bands and vague connections between clearly separated bands is avoided. The default value of the threshold is set to 0.1 which corresponds to a transmittance of $\approx 80 \%$ in the band relative to the background.

The selected regions are then Laplace filtered along the main (medial) axis. The filter size (in Figure 7) refers to the size of the Laplace filter convolution window. This second order derivative filtering leads to the detection of hills (negative areas) and valleys (positive areas) in the grey value image. All hill-points are labeled and every set of connected points forms a candidate for a band. For each band, parameters are calculated such as: area, total optical density, begin, end, and middle position relative to the top. If "Merge..." is set, the system tries to match bands on separate chromatids based on their projected begin and end locations. From this information a subset of the bands is extracted and used for classification. Athena uses the central position of the following bands:

- Darkest band on the chromosome 
- Band having the largest area

- First band on the p-terminal

- Last band on the q-terminal

- First band after the centromere position on the q-terminal.

After the rotation, the centromere localization, and the band measurement, the seven features - length, CI and the five band positions - are assembled (see Figure 8) and stored on disk in the same file that contains the experiment description as shown in Figure 3. The user may examine the data by summoning the Features window from the Windows menu.

\begin{tabular}{|c|c|c|c|c|c|c|c|}
\hline 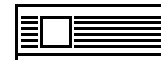 & 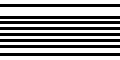 & 昰 der & $0-$ List & ade & & 春 & 昰 \\
\hline otj j\# & Iength & CIndex & firste & lastB & LargeB & cente & \\
\hline 1 & 480 & 441 & 166 & 843 & 160 & 562 & \\
\hline 2 & 380 & 196 & 296 & 763 & 763 & 236 & \\
\hline 3 & 360 & 162 & 138 & 736 & 389 & 389 & \\
\hline 4 & 420 & 325 & 226 & 761 & 226 & 464 & \\
\hline 5 & 580 & 443 & 181 & 767 & 767 & 560 & \\
\hline 6 & 320 & 370 & 218 & 750 & 484 & 484 & \\
\hline 7 & 530 & 355 & 169 & 811 & 518 & 518 & \\
\hline 8 & 360 & 193 & 375 & 750 & 375 & 375 & \\
\hline 9 & 320 & 371 & 484 & 687 & 687 & 484 & \\
\hline 10 & 450 & 375 & 133 & 755 & 755 & 544 & \\
\hline 11 & 440 & 334 & 204 & 818 & 489 & 489 & \\
\hline 12 & 550 & 320 & 200 & 836 & 836 & 390 & \\
\hline 13 & 250 & 150 & 560 & 560 & 560 & 560 & \\
\hline 14 & 690 & 500 & 181 & 87 & 326 & 659 & \\
\hline 15 & 200 & 266 & 700 & 700 & 700 & 700 & \\
\hline
\end{tabular}

Figure 8: The measurements per chromosome are displayed and stored in a list mode. Object number refers to the order in which the chromosomes were found in the image and not to their class. The positions of the bands are relative to a normalized length of 1000 per chromosome. Thus object \#1 has a last band at $84.3 \%$ of the distance from the top of the chromosome. Six of the seven measurements are shown here. The last measurement is seen by "scrolling" to the right.

While the features used here are intuitive and give satisfactory classification results (see Results below), it is a simple matter to substitute another set of feature measures such as the weighted density distribution measures of Granum [1981]. In a similar fashion other techniques can be used to determine the band positions such as the non-linear Laplace filters [Van Vliet et al, 1988] instead of the conventional Laplace filters.

\section{Classification}

The final phase of processing is the classification of the chromosomes on the basis of the measured features. Two different windows (as indicated in Figure 3) are associated with 
this task. The first window, Classification Procedure (see Figure 9), allows the user to indicate the name of the database that will serve as the standard for classification, the training set, the name of the database that can be built as a given metaphase is classified, and some parameters associated with the classification algorithms.

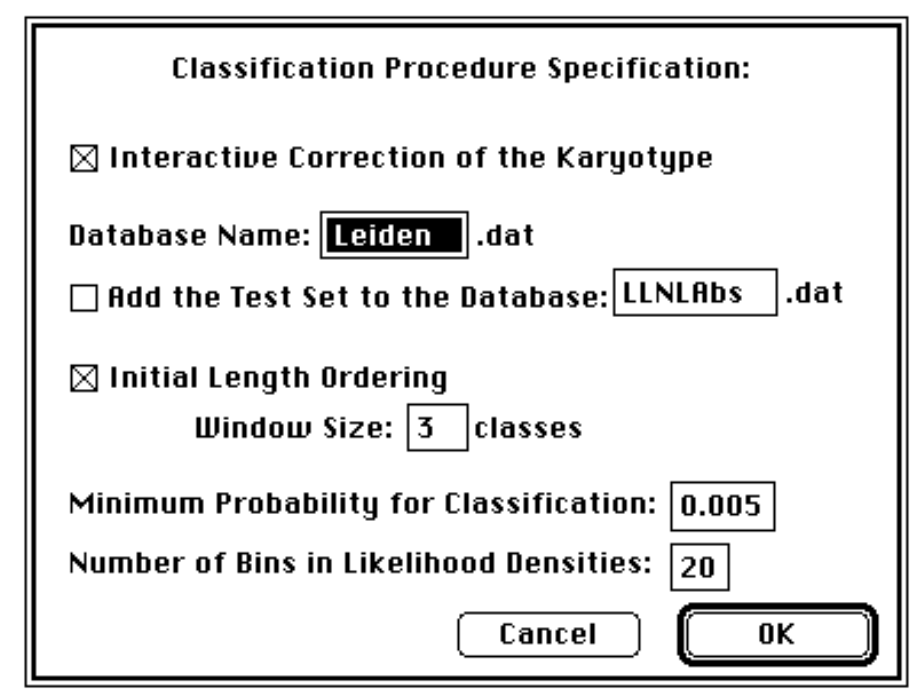

Figure 9: The first window, Classification Procedure, associated with the classification phase. The various parameters are discussed in the text.

Before classification we have measured the feature vector, $\mathbf{x}$, for each unclassified chromosome. Further, we know the estimated frequency distribution of a feature vector for each of the 24 chromosome classes $\mathbf{p}\left(\mathbf{x} \mid \omega_{\mathbf{j}}\right)$ (from the training database, e.g. Leiden.dat) and the a priori probability for a chromosome to belong to a class $\mathrm{j}, \mathbf{P}\left(\omega_{\mathbf{j}}\right)$. The $a$ priori probability is based upon biological information. (A healthy human being has 22 homologous pairs and 2 sex chromosomes). This information offers the opportunity to use the non-parametric Bayes' rule to calculate the a posteriori probability $\mathbf{P}\left(\omega_{\mathbf{j}} \mid \mathbf{x}\right)$ that each individual chromosome belongs to class $\omega_{\mathbf{j}}$ :

where

$$
P\left(\omega_{j} \mid x\right)=\frac{p\left(x \mid \omega_{j}\right) P\left(\omega_{j}\right)}{p(x)}
$$

$$
\mathrm{p}(\mathrm{x})=\sum_{\mathrm{j}=1}^{24} \mathrm{p}\left(\mathrm{x} \mid \omega_{\mathrm{j}}\right) \mathrm{P}\left(\omega_{\mathrm{j}}\right)
$$

This rule can be shown to produce, on average, the classification with the minimum number of errors.

For practical reasons we assume that the features are independent, so equation $4 \mathrm{a}$ can be rewritten as: 


$$
\mathbf{P}\left(\omega_{\mathrm{j}} \mid \mathbf{x}\right)=\frac{\prod_{n=1}^{7} \alpha_{\mathrm{n}} \mathrm{p}\left(\mathrm{x}_{\mathrm{n}} \mid \omega_{\mathrm{j}}\right) \mathbf{P}\left(\omega_{\mathrm{j}}\right)}{\mathbf{P}(\mathbf{x})}
$$

where $x_{n}$ is an element of the feature vector $\mathbf{x}$, that is, one of the seven measurements and $\left\{\alpha_{n} \mid n=1, \ldots, 7\right\}$ are weighting coefficients (see Figure 10.) For more information about the Bayes' rule see Duda and Hart [1973].

\subsection{Estimating the frequency distributions}

The frequency distributions (or class conditional probability density functions) are estimated from a learning set of patterns (database) with known class membership. Because the band features used are non-parametric, the problem of estimating $\mathbf{p}\left(\mathbf{x} \mid \omega_{\mathbf{j}}\right)$ cannot be reduced to the problem of estimating the parameters of a distribution such as the mean and standard deviation of a hypothesized Gaussian.

Athena uses the histogram approach to transform a data set into a probability density function. The range of each feature $\mathbf{x}_{\mathbf{i}}$ of vector $\mathbf{x}$ is divided into a fixed number of equal intervals or bins. The number of data points falling into each bin is counted and forms the basis for the probability estimate. The number of bins strongly depends on the size of the learning set and the underlying distribution. For Gaussian distributions the number of bins should be about $\sqrt{N}$ (if $N$ is the size of the learning set). Because the distribution of each chromosome's length is approximately Gaussian and fills about one third of the total range, $3 \sqrt{N}$ is a good estimate for the total number of bins to use. As an example, if we have 40 chromosomes per class, then the number of bins should be about $3 \sqrt{40} \approx 20$ (see Figure 9).

\subsection{Context-sensitive classification}

After computing the a posteriori probabilities for each chromosome a context sensitive classifier assigns the chromosomes to the possible classes. It is assumed that the metaphase contains two copies of each autosome and two sex chromosomes, $\mathrm{xx}$ for females and xy for males. An exception is made when there is an abnormal number of chromosomes in the cell, e.g. Down's syndrome. The classification is a two-step process.

In the first step, all chromosomes are ordered in decreasing length, building a list with the longest chromosomes first. The chromosome classes are also ordered in a list of decreasing length: 1 through 7, x, 8 through 22, and y. These two lists are then associated with each other. The first and second chromosomes from the length list with chromosome class 1, the next two chromosomes with chromosome class 2 and so forth.

After initial classification based upon length, we assign cost factors to the surrounding classes that make a transfer to another class over longer distances less likely. Distance is 
defined as the absolute difference in class number between start class and end class. Thus moving a chromosome from class 2 to class 6 is a distance of 4 . Athena (in the current version) assigns no cost $(\mathrm{C}=0)$ to classes with a distance smaller than or equal to a predefined window size (see Figure 9) from the current class leaving the a posteriori probabilities $\mathbf{P}\left(\omega_{\mathbf{j}} \mid \mathbf{x}\right)$ for $\mathbf{j}$ in (class-window) $\leq \mathbf{j} \leq$ (class+window) unchanged. An infinite cost $(\mathrm{C}=\infty)$ is assigned to all classes with a distance larger than the window size from the current class making the a posteriori probabilities $\mathbf{P}\left(\omega_{\mathbf{j}} \mid \mathbf{x}\right)$ zero for all $\mathrm{j}$ in the intervals: $\mathbf{0} \leq \mathbf{j}<$ (class-window) and (class+window) $<\mathbf{j} \leq \mathbf{2 4}$ with (class-window) $\geq$ 0 and $($ class+window $) \leq 24$.

All chromosomes are initially attached to the class with the highest a posteriori probability, still available, according to the Bayes' rule. The limit of two chromosomes per class is taken into account resulting in transfers where more than two chromosomes per class are found. This procedure continues until all chromosomes are either classified or rejected. A chromosome will be rejected if its a posteriori probability for the remaining classes is lower than a given minimum probability threshold (see Figure 9).

The second window, accessed through Features for Classification (see Figure 10), allows the user to adjust weights associated with each feature.

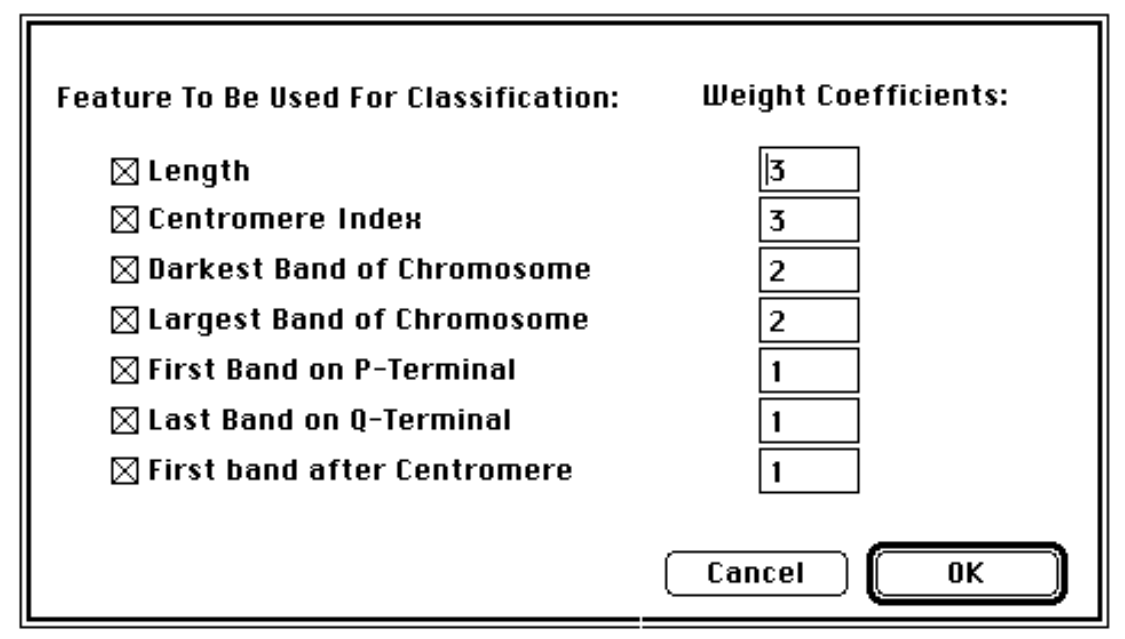

Figure 10: The second window, Features for Classification, associated with the classification phase. The values entered by the user represent the weighting coefficients $\left\{\alpha_{n}\right\}$ as used in equation 5 .

The default values used in this window were chosen for standard metaphase spreads as obtained through amniocentesis and Giemsa staining. Of particular importance in both of the windows associated with the classification phase is the ability to construct a database as well as a classifier that is "tuned" to the procedures and material within a given laboratory environment. That is, Athena can be used to construct a database de novo. 


\section{Results}

In this section we present some of the results achieved with the techniques incorporated in Athena. Some of these results - such as the centromere detection accuracy and the classification accuracy - were generated in previous studies. The procedures, however, have been incorporated unchanged into this package and thus are representative of the results that can be achieved.

\subsection{Centromere location}

In this comparison we look at five techniques for determining the position of the centromere of banded chromosomes. The first technique is based upon the smallest distance across the chromosome [Visser, 1981]. The second technique uses the maximum deviation from the convex hull of the chromosome to the chromosome contour itself [Piper, 1981]. The third technique uses a polynomial fit to the width profile of the chromosome to find the centromere [Van Zee, 1974]. The profile is filtered and the deepest minimum used as the centromere. If no clear minimum exists - as for example in many acrocentric chromosomes - the inflection point is used to determine the centromeric position. The fourth technique, based upon the work of Lucas et al [1983], uses the difference between a "standard" width profile and the measured profile. The last method, the convex profile technique is based upon the deviation between the width profile and its convex hull [Piper et al, 1980].

In all of the comparisons, the chromosomes were first rotated and straightened (after Groen and van der Ploeg, 1979]. The details of the entire experiment are described in De Muinck Keizer [1984]. The results are shown in Table I.

In a prior study [de Muinck Keizer et al, 1983], an additional technique based upon the measurement of local curvature [Gallus, 1970] was examined. This technique led to an average accuracy of $15 \%$ and was thus discarded. The shortest distance method thus provides a significant improvement in the correct identification of the centromere position when compared to the other techniques. Its performance, however, remains below the 90\% level. This indicates that, on the average, at least five chromosomes per metaphase would have to be interactively corrected. Whether this is acceptable may depend upon the nature of the user interface provided for the correction procedure. 
Table I: Accuracy of the five centromere location schemes described in the text. 924 chromosomes [Van der Ploeg et al, 1974] were used in this study. A deviation of more than one pixel from the position indicated by the "expert" was considered as an error in centromere location.

\begin{tabular}{|c|c|c|c|c|c|}
\hline $\begin{array}{c}\text { Chromosome } \\
\text { Number }\end{array}$ & $\begin{array}{c}\text { Shortest } \\
\text { Distance }\end{array}$ & $\begin{array}{c}\text { Convex } \\
\text { Hull }\end{array}$ & $\begin{array}{c}\text { Width } \\
\text { Profile (1) }\end{array}$ & $\begin{array}{c}\text { Width } \\
\text { Profile (2) }\end{array}$ & $\begin{array}{c}\text { Convex } \\
\text { Profile }\end{array}$ \\
\hline $\mathbf{1}$ & $76.5 \%$ & $52.9 \%$ & $73.5 \%$ & $64.7 \%$ & $85.3 \%$ \\
$\mathbf{2}$ & $80.0 \%$ & $71.4 \%$ & $77.1 \%$ & $62.9 \%$ & $91.4 \%$ \\
$\mathbf{3}$ & $92.3 \%$ & $43.6 \%$ & $74.4 \%$ & $59.0 \%$ & $78.9 \%$ \\
$\mathbf{4}$ & $100.0 \%$ & $72.1 \%$ & $83.7 \%$ & $69.8 \%$ & $90.5 \%$ \\
$\mathbf{5}$ & $95.6 \%$ & $73.3 \%$ & $95.6 \%$ & $44.4 \%$ & $91.1 \%$ \\
$\mathbf{6}$ & $89.2 \%$ & $75.5 \%$ & $83.8 \%$ & $86.5 \%$ & $91.9 \%$ \\
$\mathbf{7}$ & $87.8 \%$ & $82.9 \%$ & $90.2 \%$ & $87.8 \%$ & $87.8 \%$ \\
$\mathbf{8}$ & $92.3 \%$ & $97.4 \%$ & $97.4 \%$ & $76.9 \%$ & $97.4 \%$ \\
$\mathbf{9}$ & $81.1 \%$ & $78.4 \%$ & $94.6 \%$ & $81.1 \%$ & $89.2 \%$ \\
$\mathbf{1 0}$ & $89.7 \%$ & $92.3 \%$ & $82.1 \%$ & $74.4 \%$ & $87.2 \%$ \\
$\mathbf{1 1}$ & $78.6 \%$ & $73.2 \%$ & $85.7 \%$ & $61.9 \%$ & $76.2 \%$ \\
$\mathbf{1 2}$ & $83.3 \%$ & $69.0 \%$ & $83.3 \%$ & $73.8 \%$ & $83.3 \%$ \\
$\mathbf{1 3}$ & $95.3 \%$ & $92.1 \%$ & $41.9 \%$ & $83.7 \%$ & $66.7 \%$ \\
$\mathbf{1 4}$ & $97.5 \%$ & $72.5 \%$ & $17.5 \%$ & $85.0 \%$ & $52.6 \%$ \\
$\mathbf{1 5}$ & $97.3 \%$ & $92.1 \%$ & $34.2 \%$ & $92.1 \%$ & $72.7 \%$ \\
$\mathbf{1 6}$ & $83.3 \%$ & $88.4 \%$ & $86.0 \%$ & $76.7 \%$ & $100.0 \%$ \\
$\mathbf{1 7}$ & $82.1 \%$ & $71.8 \%$ & $35.9 \%$ & $84.6 \%$ & $82.1 \%$ \\
$\mathbf{1 8}$ & $92.5 \%$ & $92.7 \%$ & $36.6 \%$ & $92.7 \%$ & $95.1 \%$ \\
$\mathbf{1 9}$ & $53.5 \%$ & $82.9 \%$ & $48.8 \%$ & $67.4 \%$ & $83.3 \%$ \\
$\mathbf{2 0}$ & $69.2 \%$ & $86.5 \%$ & $74.4 \%$ & $69.2 \%$ & $94.9 \%$ \\
$\mathbf{2 1}$ & $86.8 \%$ & $39.4 \%$ & $34.2 \%$ & $71.1 \%$ & $50.0 \%$ \\
$\mathbf{2 2}$ & $62.5 \%$ & $63.9 \%$ & $57.5 \%$ & $35.0 \%$ & $61.8 \%$ \\
$\mathbf{X}$ & $96.6 \%$ & $82.2 \%$ & $100.0 \%$ & $89.7 \%$ & $96.6 \%$ \\
$\mathbf{Y}$ & $80.0 \%$ & $80.0 \%$ & $40.0 \%$ & $80.0 \%$ & $60.0 \%$ \\
$\mathbf{A v e r a g e}$ & $\mathbf{8 5 . 1 \%}$ & $\mathbf{7 6 . 1 \%}$ & $\mathbf{6 7 . 9 \%}$ & $\mathbf{7 3 . 8 \%}$ & $\mathbf{8 1 . 9 \%}$ \\
\hline
\end{tabular}

\subsection{Classification accuracy}

The complete procedure for classification described above has been tested on the same set of 924 chromosomes used for the centromere study. The test set was identical to the learning set because of the relatively small number of chromosomes per class $(\approx 20)$. The results are shown in Table II together with a comparison to Granum's WDD functions [Granum, 1981] on the same chromosomes.

Table II: Results of the classification of 924 chromosomes. In this experiment the test set equalled the learning set [ten Kate, 1983] for both classification techniques.

\begin{tabular}{|l|c|c|}
\hline $\begin{array}{c}\text { Leiden } \\
\text { Data Set }\end{array}$ & $\begin{array}{c}\text { Laplace/Band } \\
\text { Descriptors }\end{array}$ & $\begin{array}{c}\text { WD } \\
\text { Fuhctions }\end{array}$ \\
\hline Error rate & $4.0 \%$ & $4.1 \%$ \\
Rejects & $0.0 \%$ & $1.6 \%$ \\
\hline
\end{tabular}


Further, the two techniques - Laplace/band description versus WDD functions - were also compared on a much larger data set obtained from Lundsteen [1980]. The results are given in Table III.

Table III: Results of the classification experiment on the Lundsteen et al [1980] data set.

\begin{tabular}{|l|c|c|}
\hline $\begin{array}{c}\text { Copenhagen } \\
\text { Data Set }\end{array}$ & $\begin{array}{c}\text { Laplace/Band } \\
\text { Descriptors }\end{array}$ & $\begin{array}{c}\text { WD } \\
\text { Fuhctions }\end{array}$ \\
\hline Error rate & $11.5 \%$ & $2.1 \%$ \\
Rejects & $0.0 \%$ & $0.1 \%$ \\
\hline
\end{tabular}

In the Laplace/Band Descriptors technique, 7284 chromosomes were classified and the test set did not equal the learning set [ten Kate, 1983]. Bent chromosomes were classified but not straightened. In the WDD technique, 6985 chromosomes were classified and the test set did equal the learning set. Further, bent chromosomes were excluded from the classification procedure.

The use of the learning set as the test set in the testing of the WDD function approach and the exclusion of bent chromosomes mean that the value of $2.1 \%$ must be considered as highly optimistic. It is not possible for us to conclude that the technique we have implemented - a context sensitive classifier based upon bands identified by a form of Laplace filtering - is better than the WDD classifier. We can conclude, however, that it offers a reasonable accuracy. Further, the description developed by this classifier is much closer to the verbal description offered by cytogeneticists and embodied in the Paris convention [1971].

\section{Conclusions}

We have described in this article a software system for the (semi-)automatic analysis of metaphase spreads based upon a Macintosh II personal computer. This system takes full advantage of the hardware facilities in the computer: the 32 bit address space, the high speed disk, the floating point co-processor (required for chromosome rotation and straightening), the 8-bit deep display for color and grey levels, and the mouse-based user interface. As an indication of performance, the $512^{2}$ digitized metaphase image shown in Figure 11a and as a karyogram in Figure $11 \mathrm{~b}$ requires a total of three minutes for processing. Less than fifty percent of this time represents "hands-off" automatic processing and the rest is used for the interactive correction of the segmentation and the final classification. In testing the newest version of Athena on a variety of metaphase spreads - including some produced in cell irradiation experiments - the automatic processing time was about 90 seconds and the time required for interaction ranged from an additional 90 seconds to an additional 270 seconds. 
Athena: A Macintosh-Based Interactive Karyotyping System
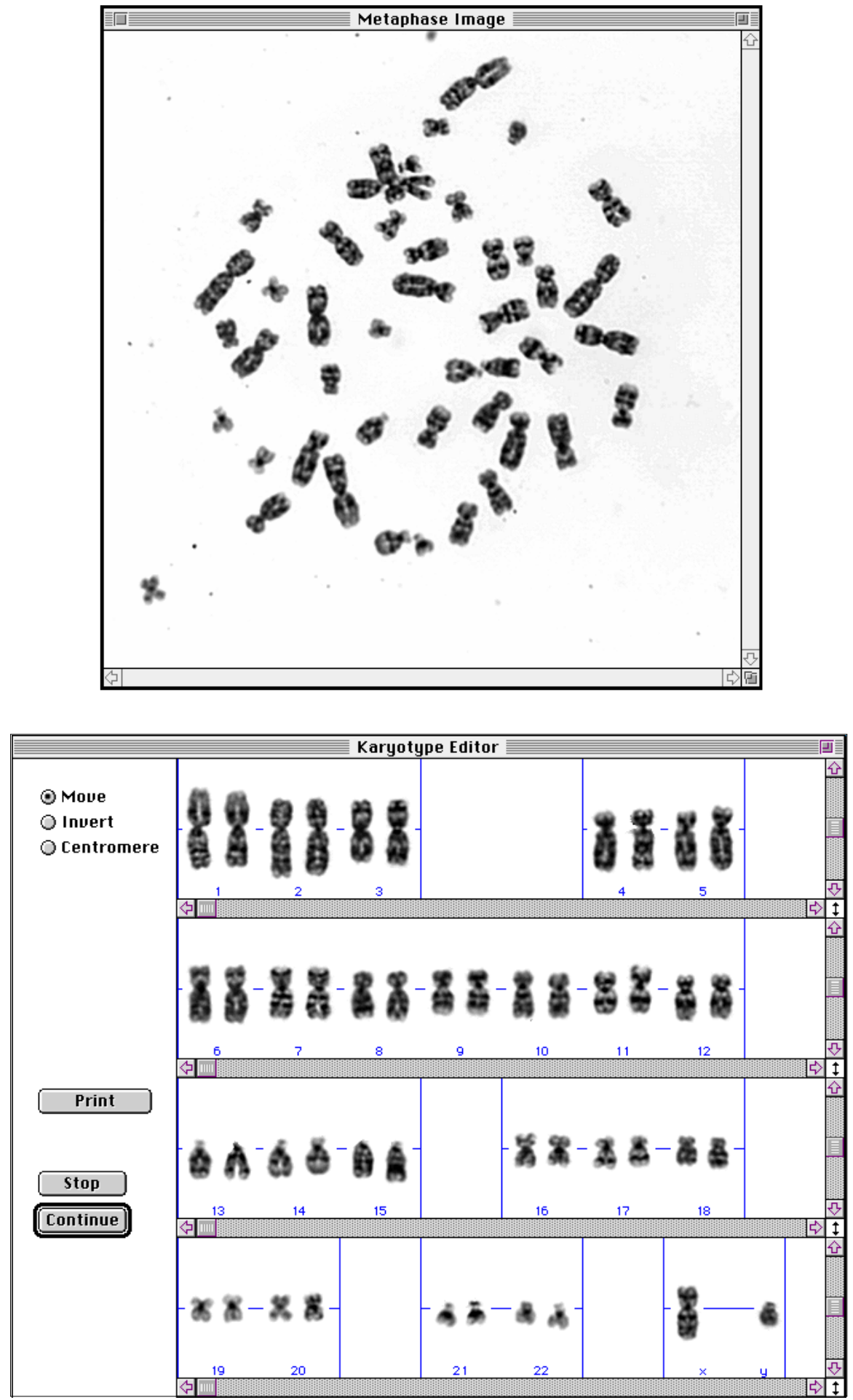

Figure 11. 
Because of the flexibility of this system - the ability to build a database with "fine-tuning" of the classification, analysis and classification parameters - we feel that it should be possible to extend it easily to a variety of cytogenetic material such as corionic villi, bone marrow, and solid tumors. We look forward to reporting in the future about the developments in Athena.

Acknowledgement. This work was partially supported by the Commission of the European Communities through the Medical and Health Research Program, project number II.1.1/13. We would also like to acknowledge the help and advice of our colleagues in Leiden, Copenhagen, Edinburgh, and Livermore.

\section{References}

1. APDA - Apple Programmer's Development Association (1988), Macintosh Programmer's Workshop Reference - Version 2.0, Renton, Washington.

2. Apple, Inside Macintosh Vol. I-III, Addison-Wesley Publishing Company Inc., Reading, Massachusetts, 1985.

3. Apple, Inside Macintosh Vol. IV, Addison-Wesley Publishing Company Inc., Reading, Massachusetts, 1986.

4. Apple, Inside Macintosh Vol. V, Addison-Wesley Publishing Company Inc., Reading, Massachusetts, 1987.

5. Bishop RP and Young IT (1977), The automated classification of mitotic phase for human chromosome spreads, Journal of Histochemistry and Cytochemistry, Vol. 25:7, pp. 730-740.

6. Caspersson T, Zech L, Johansson C (1970a), Differential banding of alkylating fluorochromes in human chromosomes, Experimental Cell Research, Vol. 60, pp. 315-319.

7. Caspersson T, Zech L, Johansson C and Modest EJ (1970b), Identification of human chromosomes by DNA-binding fluorescent agents, Chromosoma, Vol. 30, p. 215.

8. de Muinck Keizer M, Groen FCA, Young IT, Smeulders AWM (1983), An objective comparison of various centromere determination techniques, Proceedings of the Vth European Chromosome Analysis Workshop, Heidelberg, West Germany.

9. de Muinck Keizer M (1984), An objective comparison of various centromere determination techniques, Master's thesis, Department of Applied Physics, Delft University of Technology, (in Dutch), (Thesis supervisors: F.C.A. Groen and I.T. Young).

10. Deriugen NG (1957), The power spectrum and correlation function of the television signal, Telecommunications, Vol. 7, pp. 1-12.

11. Duda RO and Hart PE, Pattern Classification and Scene Analysis, John Wiley - Interscience, 1973.

12. Duin RPW (1983), Interactive image processing in a multi-user environment, in: Computing Structures for Image Processing, Ed. MJB Duff, Academic Press, London, Chapter 8.

13. Gallus G and Neurath PW (1970), Improved computer chromosome analysis incorporating preprocessing and boundary analysis, Physics in Medicine and Biology, Vol. 15, pp. 435-445.

14. Granum E, Gerdes T, Lundsteen C (1981), Simple weighted density distributions, WDD's for discrimination between G-banded chromosomes, Proceedings of the IVth European Chromosome Analysis Workshop, Edinburgh. 
15. Granlund GH, Zack GW, Young IT, Eden M (1976), A technique for multiple-cell chromosome karyotyping, Journal of Histochemistry and Cytochemistry, Vol. 24:1, pp. 160-167.

16. Groen FCA, Verbeek PW, Zee GA van der, Oosterlinck A (1976), Some aspects concerning computation of chromosome banding profiles, in Proceedings of the IIIrd International Joint Conference on Pattern Recognition, Coronado, California, pp. 547-550.

17. Groen FCA and van der Ploeg M (1979), DNA cytophotometry of human chromosomes, Journal of Histochemistry and Cytochemistry, Vol. 27, pp. 435-440.

18. Groen FCA, Young IT, Ligthart G (1985), A comparison of different focus functions for use in autofocus algorithms, Cytometry, Vol. 6, pp. 81-91.

19. Krusemark S and Haralick RM (1983), An operating system interface for transportable image processing software, Computer Vision, Graphics, and Image Processing, Vol. 23, pp. 42-66.

20. Ledley RS (1964), High speed automatic analysis of biomedical pictures, Science, Vol. 146, pp. 216-223.

21. Ledley RS, Rotolo LS, Golab TJ, Jacobsen JD, Ginsberg MD, Wilson JB (1965), FIDAC Film input to digital automatic computer and associated syntax directed pattern recognition programming system, in Optical and Electro-Optical Information Processing, Ed's. Tippet JI, Beckowitz D, Clapp L, Koester C, Vanderburgh A, MIT Press, Cambridge, Mass., pp. 591631.

22. Lucas JN, Gray JW, Peters DC, Van Dilla MA (1983), Centromeric index measurement by slit-scan flow cytometry, Cytometry, Vol. 4:2, pp. 109-116.

23. Lundsteen C, Philip J, Granum E (1980), Quantitative analysis of 6985 digitized trypsin Gbanded human metaphase chromosomes, Clinical Genetics, Vol. 18, pp. 335-370.

24. Mayall BH, Carrano AV, Rowley JD (1974), DNA cytophotometry of chromosomes in a case of chronic myelogeneous leukemia, Cancer Research, Vol. 37, pp. 3590-3593.

25. Mayall BH, Carrano AV, Golbus MS, Conte FA, Epstein CJ (1977a), DNA cytophotometry in prenatal cytogenetic diagnosis, Clinical Genetics, Vol. 1, pp. 273-276.

26. Mayall BH, Carrano AV, Moore DH, Rowley JD (1977b), Quantification by DNA-based cytophotometry of the $9 q+/ 22 q$-chromosomal translocation associated with chronic myelogeneous leukemia, Cancer Research, Vol. 37, pp. 3590-3593.

27. Mayall BH, Carrano AV, Moore DH, Ashworth LK, Bennet DE, Mendelsohn ML (1984), The DNA-based human karyotype, Cytometry, Vol. 5, pp. 376-385.

28. Mendelsohn ML, Hungerford DA, Mayall BH, Perry BH, Conway TJ, Prewitt JMS (1969), Computer-oriented analysis of human chromosomes. II. Integrated optical density as a single parameter for karyotype analysis, Annals of the New York Academy of Science, Vol. 157, pp. 376-393.

29. Mendelsohn ML and Mayall BH (1972), Computer-oriented analysis of human chromosomes. III. Focus, Computers in Biology and Medicine, Vol. 2, pp. 137-150.

30. Mendelsohn ML, Mayall BH, Bogart E, Moore DH, Perry BH (1973), DNA content and DNAbased centromeric index of the 24 human chromosomes, Science, Vol. 179, pp. 1126-1129.

31. Mendelsohn ML, Bennet DE, Bogart E, Mayall BH (1974), Computer-oriented analysis of human chromosomes. IV. Deoxyribonucleic acid based centromeric index, Journal of Histochemistry and Cytochemistry, Vol. 22, pp. 554-560.

32. Neurath PW et al (1965), Human chromosome analysis by computer - an optical pattern recognition problem, Annals of the New York Academy of Science, Vol. 128, pp. 1013-1028.

33. Paris Conference (1971), Standardization in human cytogenetics, Birth Defects: Original Article Series, National Foundation - March of Dimes, New York, Vol. VIII:7. 
34. Piper J, Granum E, Rutovitz D, Ruttledge H (1980), Automation of chromosome processing, Signal Processing, Vol. 2, pp. 203-221.

35. Piper J (1981), Finding chromosome centromeres using boundary and density information, in Digital Image Processing, Ed's. J-C Simon and RM Haralick, Dordrecht, The Netherlands, pp. 511-518.

36. Ploeg M van der, Duyn P van, Ploem JS (1974), High resolution scanning densitometry of photographic negatives of human metaphase chromosomes, Parts I and II, Journal of Histochemistry and Cytochemistry, Vol. 42, pp. 9-46.

37. Preston K (1983), Progress in image processing languages, in: Computing Structures for Image Processing, Ed. MJB Duff, Academic Press, London, Chapter 13.

38. Ridler TW and Calvard S (1978), Picture thresholding using an interactive selection method, IEEE Transactions on Systems, Man and Cybernetics, Vol. SMC-8:8, pp. 630-632.

39. Schreiber WF (1956), Third-order probability distribution of television signals, IRE Transactions on Information Theory, Vol. IT-2:3, pp. 94.

40. Tamura H, Sakane S, Tomita F, Yokoya N, Kaneko M, and Sakaue K (1983), Design and implementation of SPIDER - A transportable image processing software package, Computer Vision, Graphics, and Image Processing, Vol. 23, pp. 273-294.

41. Ten Kate TK, Groen FCA, Young IT, van der Ploeg M, Pearson PL, Gerdes T, Lundsteen C (1983), The Delft classification technique on some difficult chromosome classes, Proceedings of the Vth European Chromosome Analysis Workshop, Heidelberg, West Germany.

42. Ten Kate TK (1985), Design and implementation of an interactive karyotyping program in $C$ on a Vicom digital image processor, Master's thesis, Department of Applied Physics, Delft University of Technology (Thesis supervisors: F.C.A. Groen and I.T. Young).

43. Tjio JH and Levan H (1956), The chromosome number in man, Hereditas, Vol. 42, pp. 1-6.

44. Visser RT (1981), Classification of banded chromosomes using a priori cytologic knowledge, Master's thesis, Department of Applied Physics, Delft University of Technology, (in Dutch), Master's thesis, Department of Applied Physics, Delft University of Technology (Thesis supervisor: F.C.A. Groen).

45. Vliet LJ van and Verwer BJH (1988), A contour processing method for fast binary neighborhood operations, Pattern Recognition Letters, Vol. 7, pp. 27-36.

46. Vliet LJ van, Young IT, Beckers ALD (1988), A non-linear Laplace operator as edge detector in noisy images, accepted by Computer Vision, Graphics, and Image Processing.

47. Vossepoel AM, Smeulders AWM, Van de Broek K (1979), DIODA: Delineation and feature extraction of microscopical objects, Computer Programs in Biomedicine, Vol. 10, pp. 231-244.

48. Young IT, Groen FCA, Dorst L, Geerlings AC, Jovin T, Arndt-Jovin DJ (1983), Comparative imaging and quantification of DNA species in chromosomes, Proceedings of the Vth European Chromosome Analysis Workshop, Heidelberg, West Germany.

49. Young IT and Roos R (1988), Acuity: Image analysis for the personal computer, in: Pattern Recognition in Practice, Vol. III, Ed's. ES Gelsema and LN Kanal, North Holland Press, Amsterdam.

50. Zack GW, Spriet JA, Latt SA, Granlund GH, Young IT (1976), Automatic detection and localization of sister chromatid exchanges, Journal of Histochemistry and Cytochemistry, Vol. 24:1, pp. 168-177.

51. Zee GA van (1974), Automated chromosome analysis of Feulgen stained specimens, Master's thesis, Department of Applied Physics, Delft University of Technology, (in Dutch), Master's thesis, Department of Applied Physics, Delft University of Technology (Thesis supervisor: F.C.A. Groen). 